\title{
openheart Routinely collected health data to study inherited heart disease: a systematic review (2000-2016)
}

\author{
Bianca Blanch, ${ }^{1,2}$ Joanna Sweeting, ${ }^{1,2}$ Christopher Semsarian, ${ }^{1,2,3}$ Jodie Ingles ${ }^{1,2,3}$
}

\begin{abstract}
- Additional material is published online only. To view please visit the journal (http:// dx.doi.org/10.1136/openhrt2016-000686).
\end{abstract}

To cite: Blanch B, Sweeting J, Semsarian C, et al. Routinely collected health data to study inherited heart disease: a systematic review (2000-2016). Open Heart 2017;4:e000686. doi:10.1136/ openhrt-2017-000686

Received 17 July 2017 Revised 23 August 2017 Accepted 12 September 2017

\section{(1) CrossMark}

${ }^{1}$ Agnes Ginges Centre for Molecular Cardiology, Centenary Institute, Sydney, New South Wales, Australia

${ }^{2}$ Sydney Medical School, University of Sydney, Sydney, New South Wales, Australia ${ }^{3}$ Department of Cardiology, Royal Prince Alfred Hospital, Camperdown, New South Wales, Australia

Correspondence to Dr Jodie Ingles; j.ingles@ centenary.org.au

\begin{abstract}
Objective Our understanding of inherited heart disease is predominantly based on retrospective specialised clinic cohorts, which have inherent selection bias. Populationbased routinely collected data can provide insight into unbiased, large-scale patterns of treatment and care but may be limited by the granularity of clinical information available. We sought to synthesise the global literature to determine whether we can identify patients with inherited heart diseases using routinely collected health data. Methods Medline, Embase, CINAHL, PreMEDLINE and Google Scholar citation databases were searched for relevant articles published between 1 January 2000 and 31 October 2016
\end{abstract}

Results A total of 5641 titles/abstracts were screened and 46 full-text articles were retrieved. Twelve peerreviewed, English-language manuscripts met our inclusion criteria. Studies predominantly focused on Marfan syndrome (41\%) or hypertrophic cardiomyopathy (29\%). All studies used International Classification of Disease diagnosis codes to define inherited heart disease populations; three studies also used procedure codes. Nine of the 17 definitions for inherited heart disease were repeated across studies.

Conclusions Inherited heart disease populations can be identified using routinely collected health data, though challenges relate to existing diagnosis codes. This is an underutilised resource with the potential to inform patterns of care, patient outcomes and overall disease burden.

\section{INTRODUCTION}

Inherited heart diseases include the genetic cardiomyopathies, primary arrhythmogenic disorders and familial connective tissue and valve diseases. The prevalence of inherited heart diseases ranges from 1 in 200 to 10 000 in the general population ${ }^{12}$ and most are characterised by marked clinical and genetic heterogeneity. ${ }^{3} \mathrm{~A}$ diagnosis is contingent on clinical investigations with a cardiologist; a detailed three-generation family history noting any sudden deaths or heart disease; and in some cases, genetic testing. ${ }^{4}$ Clinical and family management of inherited heart diseases are based on published guidelines. ${ }^{35-7}$ However, to date, much of this evidence is derived from clinical trials and patient registries, which have inherent selection biases. ${ }^{4}$

Routinely collected data are obtained for administrative and clinical purposes without any specific a priori research goals. ${ }^{8}$ These health data are linked, person-level and longitudinal, providing researchers with the opportunity to examine one patient's interactions with the healthcare system, for any medical reason, over the entire observation period. Routinely collected health data are also population-based, meaning they capture information for all persons receiving treatment. These strengths are particularly noteworthy when investigating rare diseases, as they may be more effective at identifying a representative sample, which increases the generalisability of findings and reduces selection biases. However, routinely collected health data typically include only a limited amount of clinical information so it is unclear whether these data alone can be used to accurately identify an inherited heart disease population. We sought to conduct a global systematic review to examine current methods of identifying patients with inherited heart diseases using routinely collected health data exclusively.

\section{METHODS}

\section{Eligible studies}

The review included English-language, peer-reviewed articles, published between 1 January 2000 and 31 October 2016, which identified patients with an inherited heart disease using routinely collected health data exclusively. We included the following inherited heart diseases: arrhythmogenic right ventricular cardiomyopathy; bicuspid aortic valve disease; Brugada syndrome; catecholaminergic polymorphic ventricular tachycardia; familial dilated cardiomyopathy; familial hypercholesterolaemia; familial restrictive cardiomyopathy; hypertrophic cardiomyopathy; long QT syndrome; left 
ventricular non-compaction and Marfan syndrome. The RECORD (REporting of studies Conducted using Observational Routinely-collected health Data) definition of routinely collected health data was used: 'data obtained for administrative and clinical purposes without specific a priori research goals'. ${ }^{8}$ All grey literature (research that is unpublished or published in a non-commercial form), government reports, case reports/studies, editorials, commentaries, letters, conference abstracts, protocols and review articles were excluded.

\section{Search strategy}

On 31 October 2016, four bibliographic databases (Medline, PreMEDLINE, EMBASE and Cumulative Index to Nursing and Allied Health Literature [CINAHL]) were searched, combining subject headings and keywords to capture relevant studies. Search terms included those related to data type (eg, hospitalisation, medical records); methodological design (eg, cohort studies, epidemiological methods) and heart disease (eg, hypertrophic cardiomyopathy, Marfan syndrome). Online supplementary table 1A-D outlines the full search strategy. Back references and citing articles (via Google Scholar) of all manuscripts included in this review were searched to identify additional relevant articles.

Abstracts and titles of all articles were screened (BB) to identify potentially relevant studies. Two reviewers (BB and JS) independently assessed each article based on a 5-item tool specifying the eligibility criteria (online supplementary figure 1). A third reviewer (JI) independently assessed any article for which consensus was not reached ( $2 \%$ of articles).

\section{Data extraction}

The following is reported for each article:

- Study details: first author surname; year of publication; publishing journal; funding source; setting; data source(s); data coverage; observation period and study objectives relating to inherited heart disease(s). We also calculated the publication lag (year of publication - final year of observation).

- Medical condition of interest: inherited heart disease(s) studies and definition using routinely collected health data, such as International Classification of Diseases (ICD) $\operatorname{code}^{9}$ and whether any family members of affected individuals were identified.

- Cohort details: cohort eligibility criteria; number of cohorts; cohort demographics including number of persons, age (median with interquartile range [IQR] or mean with standard deviation [SD]), proportion of women; and where relevant, variables used to match cohorts.

- Outcome measures: any outcome measure related to an inherited heart disease cohort. Summary statistics are shown for statistically significant results, and summaries of non-statistically significant results are provided. In studies where no statistical analyses were performed, we report the results of relevant out- come measures. We assigned each outcome measure a theme: resource utilisation (eg, length of hospital stay, use of intensive care unit); mortality; costs (eg, hospitalisation, burden to society); occurrence of other disease/condition (eg, hypertension, cardiac condition); in-hospital procedures/intervention (eg, aortoiliac dissection, cardiovascular intervention); post-event outcomes or complications (eg, post-procedural complications, aortic repair) and prescription drug use (eg, beta-blockers).

- Summary statistics: numbers with percentages or other reported statistics (eg, mean with SD; median with IQR or range; odda ratio with confidence intervals; prevalence of condition) for relevant outcome measures. Where possible, we calculated the number and/ or proportion if not provided in the original study.

- Any other relevant findings or conclusions.

- Comprehensiveness of reporting (BB only): we scored each manuscript against the Strengthening the Reporting of Observational Studies in Epidemiology (STROBE) checklist. ${ }^{11}$ The STROBE checklist is comprised of 22-items relating to the features of the scientific process that should be included in an accurate and complete report of an observational study.

Online supplementary figure 2 features the main data extraction tool.

\section{Analysis}

The study objectives and outcome measures varied across reviewed manuscripts. Therefore, it was not appropriate to use traditional meta-analysis approaches to pool individual study results. A descriptive analysis, with details of the key findings of individual studies, was provided. Summaries of study features are shown in the tables and figures. The review is consistent with A Measurement Tool to Assess Systematic Reviews (AMSTAR) and Preferred Reporting Items for Systematic Reviews and Meta-Analyses (PRISMA) guidelines $^{1213}$ (online supplementary table 2A,B).

\section{RESULTS}

\section{Studies identified}

The titles/abstracts of 5641 articles were screened, and 46 full-text manuscripts were retrieved and reviewed. Twelve manuscripts met the eligibility criteria; nine were identified from database searches and three from back reference/citing article search (figure 1). For a list of all included and excluded studies, see online supplementary tables 3 and 4 , respectively.

\section{Study features}

The studies were set in the USA (seven studies), Germany (two studies), Taiwan (one study), Hong Kong (one study), and one study did not specify the setting (table 1 ). Eight articles $(67 \%)$ were published between 2013 and 2016. For the 11 studies (92\%) that reported an observation period, the median was 8 years (range: 3-14 years) and the median publication lag was 4 years (range: $2-6$ years). One study used two data sources; the remaining 


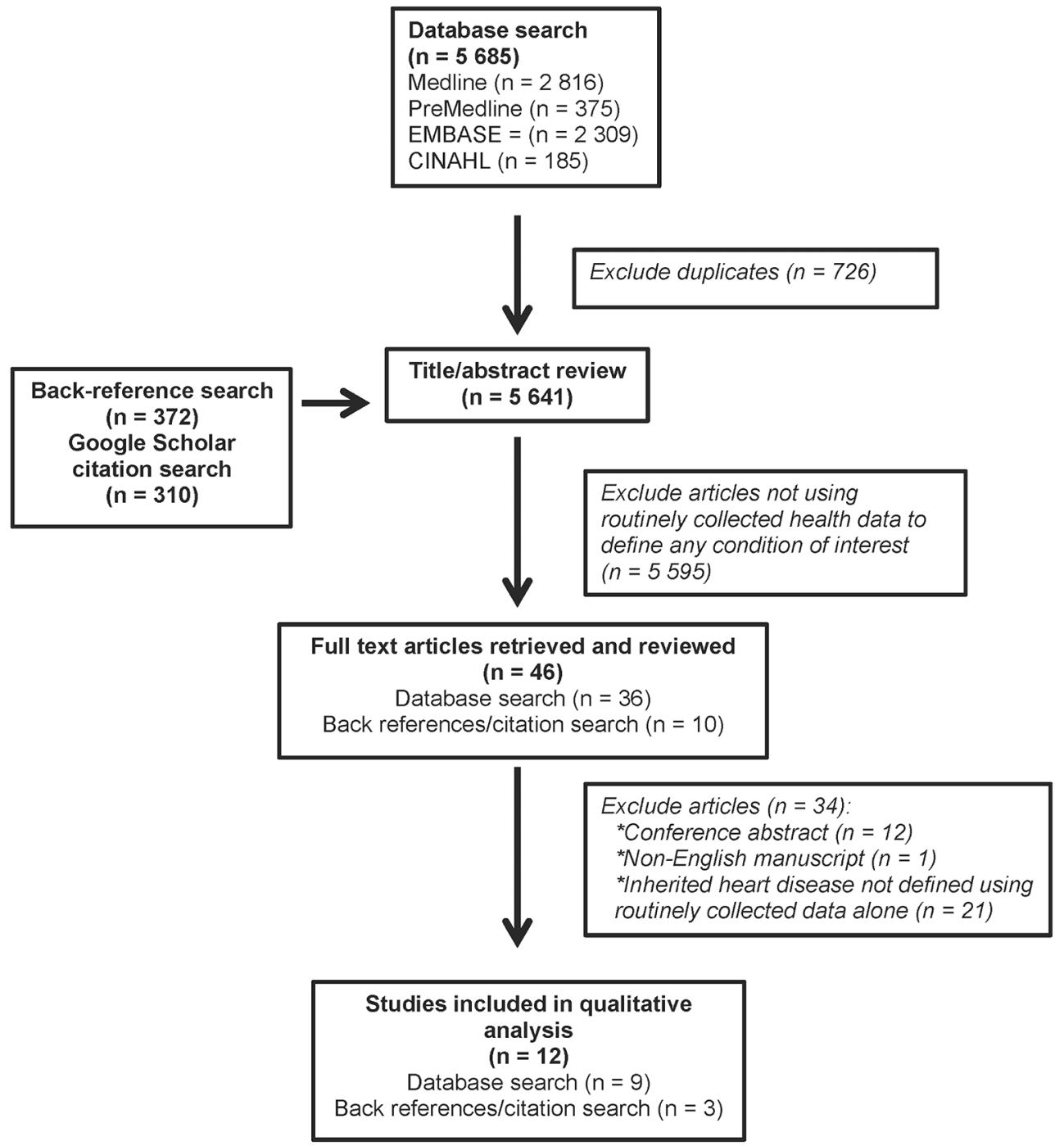

Figure 1 Flow chart of study identification and selection.

11 used one data source. Hospitalisation data were the most commonly used (eight studies [62\%]) data source. One study used medical records from two urban hospitals ${ }^{14}$; one study used state-based data ${ }^{15}$ and the remaining 10 studies $(83 \%)$ used a national dataset that covered $1 \%,{ }^{16} 9 \%,{ }^{17} 1820 \%^{19-22}$ or $100 \%^{23} 24$ of the population. Only one-quarter of studies reported a funding source, most commonly research/government grants (three studies). One study defined six inherited heart diseases; the other 11 studies focused on one disease, Marfan syndrome (seven studies [41\%]) or hypertrophic cardiomyopathy (four studies [29\%]) (table 1).

\section{Cohort characteristics}

Nine studies $(75 \%)$ reported a cohort size. Our review included 17269 persons with an inherited heart disease; 5794 persons with Marfan syndrome (range: 12-2 329) and 11475 persons with hypertrophic cardiomyopathy (range: 227-11 248). Two studies reported the number of procedures rather than people (total=1023, range: $358-665$ ). One study did not report a sample size (online supplementary table 5).
Nine studies $(75 \%)$ reported the mean age of an inherited heart disease cohort (range: 12-68 years). Marfan syndrome cohorts were younger than hypertrophic cardiomyopathy cohorts (range: $12-49$ vs 57-68, respectively). Eleven studies $(92 \%)$ reported cohort sex demographics. Of these, two studies $(17 \%)$ restricted their cohort to women. In the remaining nine studies, hypertrophic cardiomyopathy cohorts (four studies) were predominantly women (range: $53 \%-62 \%$ ), whereas Marfan syndrome cohorts (five studies) were predominantly men (female proportion range: 37\%-43\%) (online supplementary table 5).

Three studies $(25 \%)$ reported a control cohort including $7 \quad 095 \quad 507$ persons (range: 554-7 094 061). ${ }^{161719}$ Two studies matched controls to the inherited heart disease population using multiple variables including sex, age, comorbidities, pharmaceutical claims and/or year of surgical procedure. ${ }^{16} 17$

None of the studies used routinely collected data to identify family members of patients with an inherited heart disease. One study reported maternal and fetal outcomes of pregnant women with Marfan syndrome. 
Table 1 Characteristics of included studies focusing on inherited heart disease $(\mathrm{N}=12)$

\begin{tabular}{|c|c|}
\hline Study characteristic & n (\%) \\
\hline \multicolumn{2}{|l|}{ Setting } \\
\hline North America & $7(58)$ \\
\hline Europe & $2(17)$ \\
\hline Asia & $2(17)$ \\
\hline Not specified & $1(8)$ \\
\hline \multicolumn{2}{|l|}{ Year of publication } \\
\hline 2000-2004 & $1(8)$ \\
\hline 2005-2008 & $2(17)$ \\
\hline 2009-2012 & $1(8)$ \\
\hline 2013-0ctober 2016 & $8(67)$ \\
\hline \multicolumn{2}{|l|}{ Publication lag } \\
\hline $1-2$ years & $2(17)$ \\
\hline $3-5$ years & $8(67)$ \\
\hline $6-9$ years & $1(8)$ \\
\hline Not specified & $1(8)$ \\
\hline \multicolumn{2}{|l|}{ Inherited heart disease(s) of interest* } \\
\hline Marfan syndrome & $7(41)$ \\
\hline Hypertrophic cardiomyopathy & $5(29)$ \\
\hline $\begin{array}{l}\text { Arrhythmogenic right ventricular } \\
\text { cardiomyopathy }\end{array}$ & $1(6)$ \\
\hline Brugada syndrome & $1(6)$ \\
\hline $\begin{array}{l}\text { Catecholaminergic polymorphic ventricular } \\
\text { tachycardia }\end{array}$ & $1(6)$ \\
\hline Left ventricular non-compaction & $1(6)$ \\
\hline Long QT syndrome & $1(6)$ \\
\hline \multicolumn{2}{|l|}{ Data source(s)† } \\
\hline Hospitalisation data & $8(62)$ \\
\hline Health insurance & $3(23)$ \\
\hline Medical records & $2(15)$ \\
\hline \multicolumn{2}{|l|}{ Data coverage } \\
\hline National & $9(75)$ \\
\hline Single jurisdiction & $2(17)$ \\
\hline Not specified & $1(8)$ \\
\hline
\end{tabular}

*One paper defined six inherited heart diseases (denominator=17). †One paper used two data sources (denominator=13).

Compared with controls, women with Marfan syndrome required significantly more medical interventions during the birth, their fetuses were smaller and they had a higher frequency of pre-term birth. ${ }^{19}$

\section{Definition of inherited heart disease using routinely collected health data}

Seven of the 12 inherited heart diseases included in our search strategy were defined in at least one study (table 2). All studies specified ICD-9 or ICD-10 diagnosis codes to define an inherited heart disease population; three studies also used procedure codes. ${ }^{20-22}$ Across all 12 studies, there were
17 definitions of inherited heart disease. Of these, nine definitions were used in at least two studies or conditions.

\section{Marfan syndrome}

Two of the seven Marfan syndrome studies did not further detail relevant ICD-9 $\operatorname{code}(\mathrm{s}) .{ }^{1423}$ The remaining five studies had a consistent definition of Marfan syndrome based on the coding system used by the routinely collected data source. Specifically, the two German studies used the ICD-10-GM (German Modification) diagnosis code Q87.4, given as either an in-hospital diagnosis or two outpatient diagnoses within 6 months. ${ }^{17} 18$ The three studies using ICD-9-CM (Clinical Modification) all used the diagnosis code $759.82 .{ }^{19} 2425$ One of these studies required the diagnosis code to be either the primary or secondary diagnosis.

\section{Hypertrophic cardiomyopathy}

Four of the five hypertrophic cardiomyopathy studies used the ICD-9-CM diagnosis code of 425.1 to identify hypertrophic cardiomyopathy ${ }^{16}$ 20-22; one study used the ICD-9-CM code of 425.18. ${ }^{15}$ Two of the five studies used the ICD diagnosis code alone to identify patients with hypertrophic cardiomyopathy. The other three studies required the patient to have a procedure code for a septal myectomy, septal ablation or alcohol septal ablation, in addition to the ICD diagnosis code of 425.1. One of these three studies also required the ICD diagnosis of hypertrophic cardiomyopathy to be the primary diagnosis.

\section{Other inherited heart diseases}

One study defined six inherited heart diseases using ICD-9 diagnosis codes. ${ }^{15}$ In addition to HCM, these conditions include Brugada syndrome (ICD-9 code of 746.89), catecholaminergic polymorphic ventricular tachycardia (427.1) and long QT syndrome (426.82). Despite left ventricular non-compaction and arrhythmogenic right ventricular cardiomyopathy being distinct diseases, both were identified using one ICD-9 code (425.4).

\section{Prevalence of inherited heart disease}

Four Marfan syndrome studies calculated the prevalence rate, which ranged from 0.5 to $3 / 10000$ persons. ${ }^{17192324}$ One study reported 5-year age-specific prevalence, and the highest prevalence was in persons aged 15-19 years (32.3/100 000 persons) followed by 10-14 years (23.6/100 000 persons) and 20-24 years (18.6/100 000 persons). ${ }^{24}$

\section{Outcome measures}

We categorised the outcome measures into seven themes (table 3); reviewed studies reported a median of three themes (range: 1-5). The most commonly reported outcome measures were resource utilisation (seven studies); mortality (seven studies); occurrence of disease 
Heart failure and cardiomyopathies

Table 2 Definitions of identifying inherited heart disease using routinely collected health data

\begin{tabular}{|c|c|c|}
\hline Inherited heart disease & $\begin{array}{l}\text { First author surname } \\
\text { (setting) }\end{array}$ & Definition of inherited heart disease \\
\hline $\begin{array}{l}\text { Arrhythmogenic right ventricular } \\
\text { cardiomyopathy }\end{array}$ & Takiguchi (USA) $^{15}$ & ICD-9 diagnosis code of 425.4 \\
\hline Brugada syndrome & Takiguchi (USA) $)^{15}$ & ICD-9 diagnosis code of 746.89 \\
\hline $\begin{array}{l}\text { Catecholaminergic polymorphic } \\
\text { ventricular tachycardia }\end{array}$ & Takiguchi (USA) $^{15}$ & ICD-9 diagnosis code of 427.1 \\
\hline \multirow[t]{5}{*}{ Hypertrophic cardiomyopathy } & Chothani (USA) $)^{22}$ & ICD-9-CM diagnosis code of 425.1 \\
\hline & Hreybe (USA) ${ }^{16}$ & ICD-9-CM diagnosis code of 425.1 \\
\hline & $\operatorname{Kim}(U S A)^{20}$ & ICD-9-CM diagnosis code of 425.1 as primary diagnosis \\
\hline & Panaich (USA) $)^{21}$ & ICD-9-CM diagnosis code of 425.1 \\
\hline & Takiguchi (USA) $)^{15}$ & ICD-9 diagnosis code of 425.18 \\
\hline Long QT syndrome & Takiguchi (USA) $)^{15}$ & ICD-9 diagnosis code of 426.82 \\
\hline Left ventricular non-compaction & Takiguchi (USA) $)^{15}$ & ICD-9 diagnosis code of 425.4 \\
\hline \multirow[t]{7}{*}{ Marfan syndrome } & Achelrod (Germany) $)^{17}$ & $\begin{array}{l}\geq 1 \text { ICD-10-GM inpatient diagnosis of } Q 87.4 \text { or } \geq 2 \text { ICD-10-GM diagnoses } \\
\text { of Q87.4 as an outpatient within } 180 \text { days }\end{array}$ \\
\hline & Carley (not specified) ${ }^{14}$ & ICD-9 code not specified \\
\hline & Chan (Hong Kong) ${ }^{23}$ & ICD-9-CM code not specified \\
\hline & Chiu (Taiwan) ${ }^{24}$ & ICD-9-CM diagnosis code of 759.82 \\
\hline & Collins (USA) $)^{25}$ & ICD-9 diagnosis code of 759.82 as a principal or secondary diagnosis \\
\hline & Hassan $(\text { USA })^{19}$ & ICD-9 code diagnosis of 759.82 \\
\hline & Roll (Germany) ${ }^{18}$ & $\begin{array}{l}\geq 1 \text { ICD-10-GM inpatient diagnosis code of } Q 87.4 \text { OR } \geq 2 \text { outpatient } \\
\text { diagnoses within } 6 \text { months }\end{array}$ \\
\hline
\end{tabular}

ICD, International Classification of Diseases; CM, Clinical Modification; GM, German Modification

(six studies); costs (five studies) and in-hospital interventions (five studies).

Compared with a control group, persons with inherited heart disease have increased costs and risk of adverse outcomes. ${ }^{16} 1719$ For example, persons with Marfan syndrome had greater annual costs than controls, of up to $€ 2366$ for direct medical costs, $€ 5875$ for direct non-medical costs and $€ 7487$ for indirect costs. ${ }^{17}$ Furthermore, the annual personal and societal costs associated with Marfan syndrome were up to $€ 61$ million and the societal burden was up to $€ 387$ million. ${ }^{17}$ Persons with

Table 3 Summary of outcome measures examined in reviewed studies $(n=12)$

\begin{tabular}{|c|c|c|c|c|c|c|c|}
\hline $\begin{array}{l}\text { First author } \\
\text { surname }\end{array}$ & $\begin{array}{l}\text { Resource } \\
\text { utilisation }\end{array}$ & Mortality & $\begin{array}{l}\text { Occurrence of } \\
\text { other medical } \\
\text { condition }\end{array}$ & Costs & $\begin{array}{l}\text { Occurrence } \\
\text { of medical } \\
\text { intervention }\end{array}$ & $\begin{array}{l}\text { Postevent* } \\
\text { outcomes/ } \\
\text { complications }\end{array}$ & $\begin{array}{l}\text { Prescription } \\
\text { drug use }\end{array}$ \\
\hline Achelrod $^{17}$ & $\checkmark$ & & & $\checkmark$ & & & $\checkmark$ \\
\hline Carley $^{14}$ & & & $\checkmark$ & & & & \\
\hline $\mathrm{Chan}^{23}$ & & $\checkmark$ & & & $\checkmark$ & & $\checkmark$ \\
\hline $\mathrm{Chiu}^{24}$ & & $\checkmark$ & & & $\checkmark$ & & \\
\hline Chothani $^{22}$ & $\checkmark$ & $\checkmark$ & $\checkmark$ & $\checkmark$ & & $\checkmark$ & \\
\hline Collins ${ }^{25}$ & $\checkmark$ & & $\checkmark$ & $\checkmark$ & $\checkmark$ & & \\
\hline Hassan $^{19}$ & $\checkmark$ & $\checkmark$ & $\checkmark$ & & $\checkmark$ & $\checkmark$ & \\
\hline Hreybe $^{16}$ & & $\checkmark$ & $\checkmark$ & & $\checkmark$ & & \\
\hline $\mathrm{Kim}^{20}$ & $\checkmark$ & $\checkmark$ & & $\checkmark$ & & $\checkmark$ & \\
\hline Panaich $^{21}$ & $\checkmark$ & $\checkmark$ & & $\checkmark$ & & $\checkmark$ & \\
\hline Roll ${ }^{18}$ & $\checkmark$ & & & & & & \\
\hline Takiguchi $^{15}$ & & & $\checkmark$ & & & & \\
\hline Total (n, \%) & $758)$ & $7(58)$ & $6(50)$ & $5(42)$ & $5(42)$ & 4 (33) & $2(17)$ \\
\hline
\end{tabular}

${ }^{\star}$ Event defined as surgical procedure or childbirth. 
Marfan syndrome also had increased risk of maternal delivery and morbidity/mortality outcomes, most notably pneumothorax (OR 51.95, 95\% CI 6.18 to 437.10 ), aortic repair (OR 42.54, 95\% CI 3.62 to 500.33), maternal death (OR 22.38, 95\% CI 2.92 to 171.81) and use of forceps during delivery (OR $6.35,95 \%$ CI 4.10 to 9.83 ) compared with controls. ${ }^{19}$ Patients with hypertrophic cardiomyopathy had a significantly higher frequency of death $(6.7 \%$ vs $2.5 \%)$, myocardial infection $(2.2 \%$ vs $0.3 \%)$ or either of these outcomes $(8.8 \%$ vs $2.7 \%)$ after non-cardiac surgery compared with controls. ${ }^{16}$

\section{Comprehensiveness of reporting}

The median STROBE score was 22 (range: 14-28) out of a possible 36 (online supplementary table 6 ). At least 17 STROBE items were reported in $92 \%$ of studies. The methodological aspects that were the least reported include: identifying study design (reported in five studies), sources of bias (four studies), study sample size calculation (three studies), detailing number of persons eligible at each stage of study design (two studies) or addressing missing data (one study).

Nine studies were published from 2009, after the STROBE statement was published. The median STROBE score was slightly lower for studies published prior to the STROBE statement publication (median: 20; range 14-22) compared with those published afterwards (median: 22; range 17-28).

\section{DISCUSSION}

Routinely collected health data can be used to identify persons with inherited heart diseases, but these data are currently underutilised. The range of outcome measures and information extracted from only 12 studies demonstrates the vast potential of routinely collected health data to make significant inroads to better understand patterns of care, patient outcomes, burden of disease and resource utilisation. These data can also generate population-level evidence for priority research areas such as examining the natural history of disease and effectiveness of treatments in the real world. ${ }^{26}$ This is particularly important for inherited heart diseases as clinical trials are often impracticable and may under-represent certain populations, including women and older patients. ${ }^{26}$ Furthermore, routinely collected health data have the potential to complement other research efforts based around primary data collections, such as existing disease registries. As our knowledge of inherited heart diseases and patterns of inheritance increases, routinely collected health data can provide valuable insight by examining whole-of-population associations between patient factors, treatment and patient outcomes to inform clinical guidelines. Despite the challenges and shortcomings of current ICD coding, routinely collected health data provide the opportunity to examine the real-world impact of these diseases.
Globally, few studies have examined the societal burden of inherited heart diseases, an important consideration given the relatively young age of patients, inherent risk to family members and potential for the severe outcomes of heart failure and sudden cardiac death. In Marfan syndrome, the primary cost drivers were inpatient treatments, care by non-physicians, reduced work productivity and loss of production due to absence, disability or death. ${ }^{17}$ Understanding the burden of disease at a societal level allows for a global view of the overall impact of disease. In the setting of a disease such as Marfan syndrome with a population prevalence of approximately $2-3$ in $10000,{ }^{27}$ taking into consideration the younger age of patients, often being adolescents and young adults, will allow a greater appreciation of the true impact of disease.

All reviewed studies used an ICD diagnosis code to define the inherited heart disease cohort, which allows for simple cross-jurisdictional comparisons and examination of trends over time for specific conditions. The majority of studies only required one diagnosis code to define the cohort. Six of the 17 inherited heart disease definitions required additional information such as a procedure code ${ }^{20-2225}$ or multiple diagnoses within a specific time frame. ${ }^{17} 18$ The impact of these additional criteria on cohort demographics and/or outcomes is unclear. Researchers should perform sensitivity analyses and/or validation studies using various cohort definitions to ensure stricter definitions do not inadvertently impact study findings.

Importantly, the ICD diagnosis coding system does not explicitly define many rare diseases, with only 500 of the 6000 rare diseases having an ICD diagnosis code. ${ }^{28}$ For countries such as Australia using the ICD-10-AM, only three of the 12 inherited heart diseases included in our search strategy have an explicit ICD diagnosis code (table 4). The codes best matching the other nine conditions are broad descriptive diagnoses (eg, other cardiomyopathies to describe arrhythmogenic right ventricular cardiomyopathy), which encompass non-inherited heart diseases. This observation likely accounts for the focus on Marfan syndrome and hypertrophic cardiomyopathy in the reviewed studies, given that they have specific ICD diagnosis codes. The current underutilisation of routinely collected health data to identify inherited heart diseases may be due to the limitations of the current coding system, which points to a need for more explicit codes to truly realise the potential of routinely collected health data.

Despite using a comprehensive search strategy to identify relevant articles, one-quarter of reviewed studies were identified from reference and citation searches in the current study. Results were restricted to English-language manuscripts, which may have excluded relevant studies, and a journal contents search was not completed as our 12 reviewed articles were published in unique journals. Grey literature was also excluded from our search strategy. Further, identifying relevant studies was challenging as only six inherited heart diseases have been mapped to 
Table 4 List of inherited heart diseases and relevant International Statistical Classification of Diseases and Related Health Problems codes (ICD-9-AM)

\begin{tabular}{ll}
\hline Inherited heart condition & ICD diagnostic code and category \\
\hline Arrhythmogenic right ventricular cardiomyopathy & 142.8 Other cardiomyopathies \\
\hline Bicuspid aortic valve disease & Q23.83 Congenital bicuspid aortic valve \\
\hline Brugada syndrome & 149.8 Other specified cardiac arrhythmias \\
\hline Catecholaminergic polymorphic ventricular tachycardia & 147.2 Ventricular tachycardia \\
\hline Familial dilated cardiomyopathy & 142.0 Dilated cardiomyopathy \\
\hline Familial hypercholesterolaemia & E78.0 Pure hypercholesterolaemia \\
\hline Familial restrictive cardiomyopathy & 142.5 Other restrictive cardiomyopathy \\
\hline Hypertrophic cardiomyopathy & 142.1 Obstructive hypertrophic cardiomyopathy \\
& 142.2 Other hypertrophic cardiomyopathy \\
\hline Left ventricular non-compaction & Q24.89 Other specified congenital malformations of the heart \\
\hline Long QT syndrome & 142.8 Other cardiomyopathies \\
\hline Marfan syndrome & 142.9 Cardiomyopathy, unspecified \\
\hline
\end{tabular}

a subject heading in at least one of the bibliographic databases used in our search strategy. To overcome such limitations, future systematic reviews in this area would be enhanced if all inherited heart diseases were mapped to a subject heading.

\section{CONCLUSIONS}

Routinely collected data are an underutilised resource to understand clinical management and treatment issues affecting patients with inherited heart diseases. Despite some challenges, routinely collected data in this setting are able to provide evidence around a number of outcome measures and would have even greater utility if ICD codes were more explicit. While observational and registry-based studies have played a fundamental role in our understanding of inherited heart diseases to date, use of routinely collected data may provide an unbiased and global perspective on the true impact of these diseases.

Acknowledgements JS is the recipient of the Elizabeth and Henry HamiltonBrown scholarship from the University of Sydney. CS is the recipient of a National Health and Medical Research Council (NHMRC) Practitioner Fellowship (No. 1059156). $\mathrm{Jl}$ is the recipient of a National Heart Foundation of Australia Future Leader Fellowship (No. 100833). This study is funded in part by an NHMRC Project Grant (No. 1059515).

Contributors Authors made substantial contribution to the concept or design of the work (BB, CS, JI), acquisition, analysis (BB, JS, JI) and interpretation (BB, JI) of the data. Drafting of the work (BB, JS) and critical revision (JI, CS). Final approval (BB, JS, CS, JI). Agree to be accountable to all aspects of the work (BB, JS, CS, JI).

Competing interests None declared.

Provenance and peer review Not commissioned; externally peer reviewed.

Data sharing statement Any person may contact the corresponding author for access to any of the data used in the systematic review.

Open Access This is an Open Access article distributed in accordance with the Creative Commons Attribution Non Commercial (CC BY-NC 4.0) license, which permits others to distribute, remix, adapt, build upon this work non-commercially, and license their derivative works on different terms, provided the original work is properly cited and the use is non-commercial. See: http://creativecommons.org/ licenses/by-nc/4.0/

(c) Article author(s) (or their employer(s) unless otherwise stated in the text of the article) 2017. All rights reserved. No commercial use is permitted unless otherwise expressly granted.

\section{REFERENCES}

1. Semsarian C, Ingles J, Maron MS, et al. New perspectives on the prevalence of hypertrophic cardiomyopathy. J Am Coll Cardiol 2015;65:1249-54.

2. Fteropoulli T, Stygall J, Cullen S, et al. Quality of life of adult congenital heart disease patients: a systematic review of the literature. Cardiol Young 2013;23:473-85.

3. Ackerman MJ, Priori SG, Willems S, et al. HRS/EHRA expert consensus statement on the state of genetic testing for the channelopathies and cardiomyopathies. Europace 2011;13:1077-109.

4. Maron BJ. Hypertrophic cardiomyopathy: a systematic review. JAMA 2002;287:1308-20.

5. Priori SG, Wilde AA, Horie M, et al. HRS/EHRA/APHRS expert consensus statement on the diagnosis and management of patients with inherited primary arrhythmia syndromes: document endorsed by HRS, EHRA, and APHRS in May 2013 and by ACCF, AHA, PACES, and AEPC in June 2013. Heart Rhythm 2013:10:1932-63.

6. Elliott PM, Anastasakis A, Borger MA, et al. ESC Guidelines on diagnosis and management of hypertrophic cardiomyopathy: the Task Force for the Diagnosis and Management of Hypertrophic Cardiomyopathy of the European Society of Cardiology (ESC). Eur Heart J 2014:35:2733-79.

7. Gersh BJ, Maron BJ, Bonow RO, et al. ACCF/AHA guideline for the diagnosis and treatment of hypertrophic cardiomyopathy: a report of the American College of Cardiology Foundation/American Heart Association Task Force on Practice Guidelines. Developed in collaboration with the American Association for Thoracic Surgery, American Society of Echocardiography, American Society of Nuclear Cardiology, Heart Failure Society of America, Heart Rhythm Society, Society for Cardiovascular Angiography and Interventions, and Society of Thoracic Surgeons. J Am Coll Cardiol 2011;58:e212-60.

8. Benchimol El, Smeeth L, Guttmann A, et al. The reporting of studies conducted using observational routinely-collected health data (RECORD) statement. PLoS Med 2015;12:e1001885.

9. World Health Organization. Classification of Diseases (ICD). 2016 http://www.who.int/classifications/icd/en/ (accessed 15 Mar 2017).

10. von Elm E, Altman DG, Egger M, et al. The strengthening the reporting of observational studies in epidemiology (STROBE) statement: guidelines for reporting observational studies. Prev Med 2007;45:247-51. 
11. Vandenbroucke JP, von Elm E, Altman DG, et al. Strengthening the reporting of observational studies in epidemiology (STROBE): explanation and elaboration. Epidemiol 2007;18:805-35.

12. Shea BJ, Grimshaw JM, Wells GA, et al. Development of AMSTAR: a measurement tool to assess the methodological quality of systematic reviews. BMC Med Res Methodol 2007;7:10.

13. Moher D, Liberati A, Tetzlaff J, et al. Preferred reporting items for systematic reviews and meta-analyses: the PRISMA statement. Ann Intern Med 2009;151:264-9.

14. Carley ME, Schaffer J. Urinary incontinence and pelvic organ prolapse in women with Marfan or Ehlers-Danlos syndrome. Am J Obstet Gynecol 2000;182:1021-3.

15. Takiguchi M, Knight T, Nguyen TT, et al. Underdiagnosis of conditions associated with sudden cardiac death in children-is it the absence of a comprehensive screening program or a true low prevalence? Hawaii J Med Public Health 2016;75:42-5.

16. Hreybe $\mathrm{H}$, Zahid $\mathrm{M}$, Sonel $\mathrm{A}$, et al. Noncardiac surgery and the risk of death and other cardiovascular events in patients with hypertrophic cardiomyopathy. Clin Cardiol 2006;29:65-8.

17. Achelrod D, Blankart CR, Linder R, et al. The economic impact of Marfan syndrome: a non-experimental, retrospective, populationbased matched cohort study. Orphanet J Rare Dis 2014;9:90.

18. Roll K. The influence of regional health care structures on delay in diagnosis of rare diseases: the case of Marfan Syndrome. Health Policy 2012;105:119-27.

19. Hassan N, Patenaude V, Oddy L, et al. Pregnancy outcomes in Marfan syndrome: a retrospective cohort study. Am J Perinatol 2015;30:123-30.
20. Kim LK, Swaminathan RV, Looser P, et al. Hospital volume outcomes after septal myectomy and alcohol septal ablation for treatment of obstructive hypertrophic cardiomyopathy: US Nationwide Inpatient Database, 2003-2011. JAMA Cardiol 2016;1:324-32

21. Panaich SS, Badheka AO, Chothani A, et al. Results of ventricular septal myectomy and hypertrophic cardiomyopathy (from Nationwide Inpatient Sample [1998-2010]). Am J Cardiol 2014;114:1390-5.

22. Chothani A, Panaich SS, Patel N, et al. Septal ablation and hypertrophic obstructive cardiomyopathy: 7 years US experience. $J$ Interv Cardiol 2016;29:505-12.

23. Chan YC, Ting CW, Ho P, et al. Ten-year epidemiological review of in-hospital patients with Marfan syndrome. Ann Vasc Surg 2008;22:608-12.

24. Chiu HH, Wu MH, Chen HC, et al. Epidemiological profile of Marfan syndrome in a general population: a national database study. Mayo Clin Proc 2014:89:34-42.

25. Collins RT, Phomakay V, Zarate YA, et al. Impact of aortic aneurysm on hospitalizations in patients with Marfan Syndrome: a multiinstitutional study. Pediatr Cardiol 2015;36:132-9.

26. Force T, Bonow RO, Houser SR, et al. Research priorities in hypertrophic cardiomyopathy. Circulation 2010;122:1130-3.

27. Judge DP, Dietz HC. Marfan's syndrome. Lancet 2005;366:1965-76.

28. Rath A, Olry A, Dhombres F, et al. Representation of rare diseases in health information systems: the Orphanet approach to serve a wide range of end users. Hum Mutat 2012;33:803-8. 